\title{
Emitted Sound Analysis for Tool Flank Wear Monitoring using Hilbert Huang Transform.
}

\author{
J. Emerson Raja, W. S. Lim, and C. Venkataseshaiah
}

\begin{abstract}
In this paper, results of investigation on the relationship between emitted tool sound signal and tool flank wear during turning process are reported. A series of experiments were conducted in a turning machine with carbide tool insert for machining Aluminum work piece. The tool emitted sound signal with a fresh tool, a slightly worn tool with $0.2 \mathrm{~mm}$ flank wear and a severely worn tool with $0.4 \mathrm{~mm}$ flank wear were captured separately using ICP microphone. The captured sound signals were analyzed using Hilbert Huang Transform (HHT) and the instantaneous frequencies and amplitudes of the sound signal components were obtained. The RMS values of the amplitudes of the Intrinsic Mode Functions (IMF) corresponding to the three different tool sound signals were analyzed. Hilbert spectrum and marginal spectrum of selected IMFs of fresh, slightly worn and severely worn tool sound signals were obtained and analyzed. Based on the analysis, it was found that the amplitude of the IMF6, IMF7 and IMF8 components of the emitted tool sound signal increase steadily with the increasing tool flank wear. The results confirm that Hilbert-Huang Transform based emitted tool sound signal analysis can be confidently applied to tool flank wear monitoring.
\end{abstract}

Index Terms-Flank wear, Hilbert Huang transform, marginal spectrum, tool sound.

\section{INTRODUCTION}

Tool condition monitoring is necessary to obtain a good quality product. Effective tool condition monitoring system enables manufacturing processes like turning, drilling, or milling, optimized and automated significantly. Tool flank wear is the most important parameter that influences production performance mostly [1]. Tool wear monitoring is a difficult task because many machining processes are non-linear time-variant systems, which makes them difficult to model and secondly, the signals obtained from sensors are dependent on a number of other factors, such as machining conditions [2]. Indirect method of tool condition monitoring is based on the acquisition of measured values of process variables (such as cutting force, temperature, vibration, spindle motor current, acoustic emission, surface roughness and emitted sound) and the relationship between tool wear and these values. Salgado et al. [3] used feed cutting force, estimated from feed motor current and the information, extracted from sound signal, to predict the tool flank wear

Manuscript received January 27, 2012; revised March 29, 2012.

J. Emerson Raja is with Multimedia University, Melaka, Malaysia. (e-mail: emerson.raja@mmu.edu.my).

W. S. Lim is with Multimedia University, Melaka, Malaysia. (e-mail: wslim@mmu.edu.my ).

C. Venkataseshaiah is with Multimedia University, Melaka, Malaysia. (e-mail: venkataseshaiah@ mmu.edu.my ). using artificial neural network. Sadettin Orhan et al. [4] investigated the relationship between vibration and tool wear and concluded that the amplitude of the vibration increases steadily with the increasing tool wear. Ming-Chyuan et al. [5] and Alonso F.J. et al. [6] used audible sound generated from the cutting process as a source for monitoring tool flank wear during turning. A Samraj et al. [7] also used the emitted sound during turning operation to predict the tool flank wear using dynamic clustering estimation method.

Most of these methods are very sensitive to the correct choice and positioning of the sensor. They use traditional data analysis methods such as Fourier analysis which assume the signals are linear and stationary. According to Z.K. Peng et al. [8] the signal to be processed must be linear and temporarily stationary; otherwise, the resulting Fourier spectrum will make little physical sense. FFT also employs a convolution integral through which the signal is decomposed in terms of sine and cosine functions covering uniformly the whole data span. Therefore the Fourier transform represents the global rather than any local properties of the signal [9].

On the other hand, the time-frequency analysis method can generate both time and frequency information of a signal simultaneously through mapping one dimensional signal to a two-dimensional time-frequency plane. Among all available time-frequency analysis methods, the wavelet transform is considered to be one of the best. However, the wavelet transform still has some inevitable deficiencies, including the interference terms, border distortion and energy leakage, all of which will generate a lot of small undesired spikes all over the frequency scales and make the results confusing and difficult to interpret [10]. Hence, new methods are needed to analyze the data from non-linear and non-stationary processes such as drilling, turning etc.

It seems that the Hilbert-Huang Transform (HHT) has the potential of becoming a perfect tool for analyzing non-stationary and nonlinear data [11] which is derived from the principles of Empirical Mode Decomposition (EMD) and the Hilbert Transform. It is a two step process. Firstly, EMD is applied to decompose the given signal into a set of complete and almost orthogonal components called intrinsic mode functions (IMF). Since the IMF is almost mono-component, it can determine all the instantaneous frequencies from a nonlinear and non-stationary signal. Secondly, the local energy of each instantaneous frequency component can be obtained through the Hilbert Transform [8], [9]. Yuping Zhang [12] successfully applied this new signal processing technique in analyzing vibration signals and faults diagnosis of roller bearing. In this paper, application of HHT for tool flank wear monitoring based on emitted sound during turning process of Aluminum work piece, and an improved approach 
to signal analysis are presented and discussed.

\section{RESEARCH METHODOLOGY}

Given below are the four major stages used in this research

1. Recording the emitted sound signal during turning operation with fresh, slightly and severely worn tools by using an ICP microphone.

2. Decomposing each multi-component sound signal into mono-component IMFs by using EMD.

3. Obtaining the local energy (amplitude) of each instantaneous frequency found in IMF by applying Hilbert Transform.

4. Constructing the Hilbert spectrum using all the IMFs, and the marginal spectrums on selected IMFs for final analysis.

The following sub sections explain the principle behind Hilbert Huang Transform, Empirical Mode Decomposition and Marginal Spectrum.

\section{A. Hilbert Huang Transform}

The physically meaningful way to describe the system is in terms of the instantaneous frequency, which will reveal the intra wave frequency modulations [9]. The easiest way to extract or compute the instantaneous frequency of a mono-component signal is by using Hilbert Transform. For an arbitrary signal or time series $x(t)$, its Hilbert transform $y(t)$ is defined as

$$
y(t)=\frac{p}{\pi} \int_{-\infty}^{+\infty} \frac{x(\tau)}{t-\tau} d \tau
$$

where $P$ is the Cauchy principal value of the singular transform. This function exists for all functions of class of Lebesgue spaces or $L^{P}$ [13]. Equation (1) shows that the Hilbert transform is defined as the convolution of the signal $x(t)$ with $1 / t$. Therefore, the Hilbert transform is capable of identifying the local properties of $x(t)$. Coupling the $x(t)$ and $y(t)$, we can have the analytic signal $z(t)$ of $x(t)$, as

$$
z(t)=x(t)+i y(t)=a(t) e^{i \varphi(t)}
$$

With

$$
a(t)=\left[x^{2}(t)+y^{2}(t)\right]^{1 / 2}, \varphi(t)=\arctan (y(t) / x(t)) .
$$

where $a(t)$ is the instantaneous amplitude of $x(t)$, which can reflect how the energy of the $x(t)$ varies with time, and the $\Phi(t)$ is the instantaneous phase of $x(t)$. The controversial instantaneous frequency $\omega(t)$ is defined as the time derivative of the instantaneous phase $\Phi(t)$, as follows:

$$
\omega(t)=d \varphi(t) / d(t) .
$$

Because the instantaneous frequency is defined through differentiation rather than integration it appears to be local and can describe intra-wave frequency modulation. Therefore (4) is useful in extracting instantaneous frequencies from any non-stationary signals. However, (4) is only valid in obtaining the instantaneous frequency of a signal in a given time frame if the signal is mono-component within the time frame. If the inspected signal is multi-component within the defined time frame, the result of the instantaneous frequency will be distorted [14]. Unfortunately, in almost all of the practical applications, the inspected signals are hardly mono-component but multi-component. Therefore to make the instantaneous frequency applicable, the key is the ability to decompose the signal into some individual mono-component signals. The Empirical Mode Decomposition provides such decomposition ability.

\section{B. Empirical Mode Decomposition}

The data, depending on its complexity, may have many different coexisting modes of oscillation at the same time. Each of these oscillatory modes is represented by an Intrinsic Mode Function (IMF) with the following definitions [9].

(a) In the whole data set, the number of extrema and the number of zero-crossings must either equal or differ at most by one, and

(b) At any point, the mean value of the envelope defined by the local maxima and the envelope defined by the local minima is zero.

To extract the IMF from a given data set, the sifting process is implemented as follows [13]. First, identify all the local maxima, and then connect all of the local maxima by a cubic spline line as the upper envelope. Then, repeat the procedure for the local minima to produce the lower envelope. The upper and lower envelopes should cover all the data between them. Their mean is designated $\mathrm{m}_{1}(\mathrm{t})$, and the difference between the data and $\mathrm{m}_{1}(\mathrm{t})$ is $\mathrm{h}_{1}(\mathrm{t})$, i.e.

$$
\mathrm{x}(\mathrm{t})-\mathrm{m}_{1}(\mathrm{t})=\mathrm{h}_{1}(\mathrm{t})
$$

The sifting process has to be repeated as many times as it is required to reduce the extracted signal to an IMF. In the subsequent sifting process steps $\mathrm{h}_{1}(\mathrm{t})$ is treated as the data; then:

$$
\mathrm{h}_{1}(\mathrm{t})-\mathrm{m}_{11}(\mathrm{t})=\mathrm{h}_{11}(\mathrm{t})
$$

where $m_{11}(t)$ is the mean of the upper and lower envelops of $h_{1}(t)$. This process can be repeated up to $k$ times; $h_{1 k}(t)$ is then given by

$$
h 1_{(k-1)}(t)-m_{1 k}(t)=h_{1 k}(t)
$$

After each processing step, checking must be done on whether the number of zero crossings equals the number of extrema. The resulting time series is the first IMF, and then it is designated as $\mathrm{c}_{1}(\mathrm{t})=\mathrm{h}_{1 \mathrm{k}}(\mathrm{t})$. The first IMF component from the data contains the highest oscillation frequencies found in the original data $x(t)$.

This first IMF is subtracted from the original data, and this difference, is called a residue $r_{1}(t)$ by:

$$
x(t)-c_{1}(t)=r_{1}(t)
$$

The residue $r_{1}(t)$ is taken as if it was the original data and we apply to it again the sifting process. The process of finding more intrinsic modes $c_{i}(t)$ continues until the last mode is found. The final residue will be a constant or a monotonic function.

$$
x(t)=\sum_{j=1}^{n} c_{j}(t)+r_{n}(t)
$$


Thus, one achieves a decomposition of the data into n-empirical IMF modes, plus a residue, $r_{n}(t)$, which can be either the mean trend or a constant.

\section{Marginal Spectrum}

Equation (2) enables us to represent the amplitude and the instantaneous frequency, in a three-dimensional plot, in which the amplitude is the height in the time-frequency plane. This time-frequency distribution is designated as the Hilbert spectrum $H(\omega, t)$ :

$$
H(\omega, t)=\operatorname{Re} \sum_{i=1}^{n} a_{i}(t) e^{j \int w_{i}(t) d t}
$$

With the Hilbert spectrum defined, the marginal spectrum, $h(\omega)$, can be defined as

$$
h(\omega)=\int_{0}^{T} H(\omega, t) d t
$$

where $\mathrm{T}$ is the total data length. The Hilbert spectrum offers a measure of amplitude contribution from each frequency and time, while the marginal spectrum offers a measure of the total amplitude contribution from each frequency [9], [12]. Therefore, local marginal spectrum of each IMF component is given as

$$
h_{i}(\omega)=\int_{0}^{T} H_{i}(\omega, t) d t
$$

The local marginal $h_{i}(\omega)$ spectrum offers a measure of the total amplitude contribution from the frequency. According to marginal spectrum, the characteristic amplitude of the tool flank wear can be easily recognized and thereby the condition of the tool wear can easily be determined.

\section{EXPERIMENTAL SET-UP}

The photography of actual experimental set-up is shown in Fig. 1. In the present experiment, the noise due to the machining was recorded by a ICP microphone, and in contrast with the accelerometer, the position of the microphone can be arbitrary [15]. A PCB 130D20 microphone is kept in a stand and facing towards the tool tip to capture the emitted sound. The microphone is connected to the computer through a specially designed signal conditioner. GoldWave software is used to record the captured sound with sampling frequency set to $44100 \mathrm{~Hz}$. A series of machining experiments were conducted on a Turning machine(XLTURN from MTAB) with Carbide Insert and aluminum work piece of $38 \mathrm{~mm}$ diameter. The details of the experiments and the various cutting conditions used are given in Table 1. First, the free running sound for the spindle rotational speed of $400 \mathrm{rev} / \mathrm{min}$ was recorded, without the machining operation. Keeping the spindle rotational speed at $400 \mathrm{rev} / \mathrm{min}$, the sound emitted due to machining with a fresh tool was recorded separately for the depth of cut of $0.5 \mathrm{~mm}$. This recording process was repeated separately for slightly worn tool with $0.2 \mathrm{~mm}$ flank wear and severely worn tool with $0.4 \mathrm{~mm}$ flank wear [16]. The 12 second long sound signal is split into 12 one second sound signals for the subsequent signal processing using HHT. Each one second signal contains 44100 sampling data. The matlab waveread function was used to digitize the sound signals.

TABLE I: THE DETAILS OF EXPERIMENT.

\begin{tabular}{|c|l|c|c|c|c|}
\hline \multirow{2}{*}{$\begin{array}{l}\text { Exp } \\
\text { No. }\end{array}$} & Name & $\begin{array}{l}\text { Farious cutting conditions } \\
\text { wear } \\
(\mathrm{mm})\end{array}$ & $\begin{array}{c}\text { Spindle } \\
\text { speed } \\
(\mathrm{rev} / \mathrm{min})\end{array}$ & $\begin{array}{l}\text { Feed } \\
\text { Rate } \\
(\mathrm{mm} / \mathrm{m})\end{array}$ & $\begin{array}{l}\text { Depth } \\
\text { of cut } \\
(\mathrm{mm})\end{array}$ \\
\hline 1 & Free run & NA & 400 & 30 & NA \\
\hline 2 & Fresh & 0.0 & 400 & 30 & 0.5 \\
\hline 3 & $\begin{array}{l}\text { Slightly } \\
\text { worn }\end{array}$ & 0.2 & 400 & 30 & 0.5 \\
\hline 4 & $\begin{array}{l}\text { Severely } \\
\text { worn }\end{array}$ & 0.4 & 400 & 30 & 0.5 \\
\hline
\end{tabular}

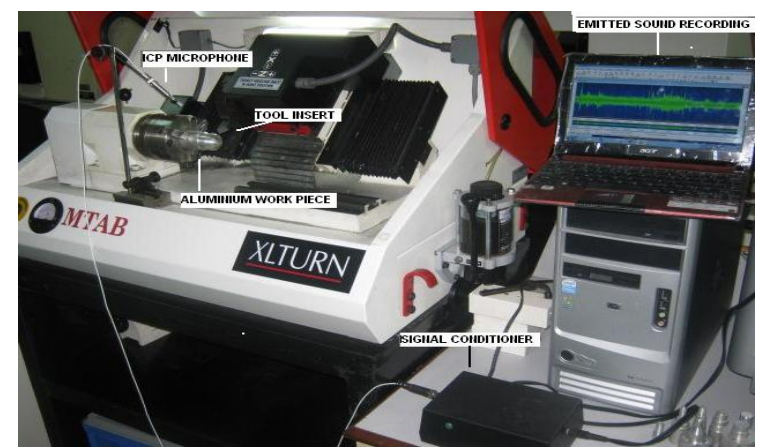

Fig. 1.The experimental setup.

\section{RESULTS AND DISCUSSION}

Fig. 2 is the plot of the original multi-component sound signal of the severely worn tool. It contains various components such as sound emitted from machine rotational parts and the much needed sound emitted due to the contact between the tool flank face and the surface of the work piece. In addition to these components, it also contains the harmonics of the fundamental signals of this kind. In order to extract the features that reveal the true nature of the tool this multi-component signal is decomposed into several mono-component signals, the IMFs, using EMD. The result of the EMD on severely worn tool sound signal is shown in the form of 15 IMFs and the residue in Fig. 3.

The Hilbert transform was then applied on the IMFs to obtain the instantaneous frequencies and amplitudes. The Hilbert spectrum, the amplitude plot on time-frequency domain, is then generated for fresh, slightly and severely worn tool sound signals. The Hilbert spectrums, shown in Fig. 8, Fig. 9, and Fig. 10 reveal the correlation between the emitted sound amplitude and the tool wear, i.e. amplitude is increasing as the wear is developing. Amplitude values around $0.5,1.0$ and 1.5 are found for fresh, slightly worn and severely worn tool respectively.

RMS amplitude value of each IMF for free run, fresh tool, slightly and severely worn tool sound signals were calculated and line graphs were generated in Fig. 4 to compare these RMS amplitude values. It is evident from Fig. 4 that IMF6, IMF7 and IMF8 are representing the sound signal emitted from the tool insert due to the contact of the tool flank face with the surface of the work piece. Out of these three IMFs, more energy in the form of RMS amplitude is found in IMF7, hence it is appropriate to perform further marginal spectral analysis on IMF7. The marginal spectrum of IMF7 for fresh, 
slightly and severely worn tool sound signals are shown in Fig. 5, Fig. 6, and Fig. 7 respectively. The maximum amplitude of sound emitted with the fresh, slightly and severely worn tool bit insert is measured as $0.997,1.514$ and 1.807 respectively. These findings show that the amplitude of the tool sound increases steadily with the increasing tool flank wear. Similar results were also obtained in our previous work where the Steel work piece instead of Aluminum was used [13]. This relationship is sufficient to train a suitable neural network to classify the state of the wear.

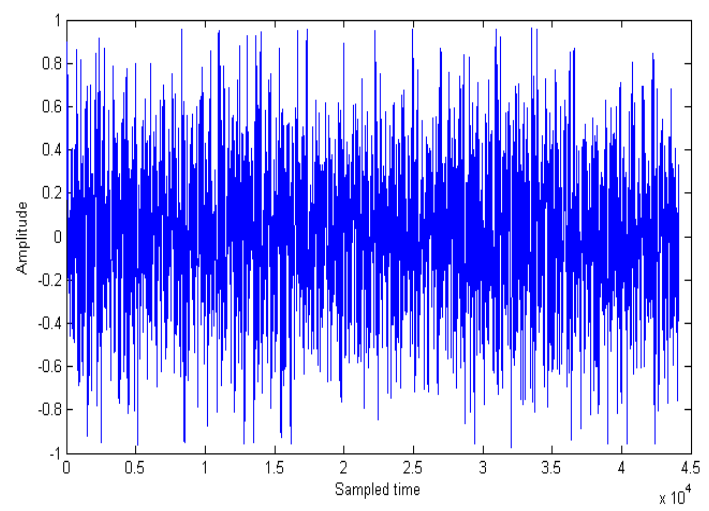

Fig. 2. The original multi-component sound signal of worn tool

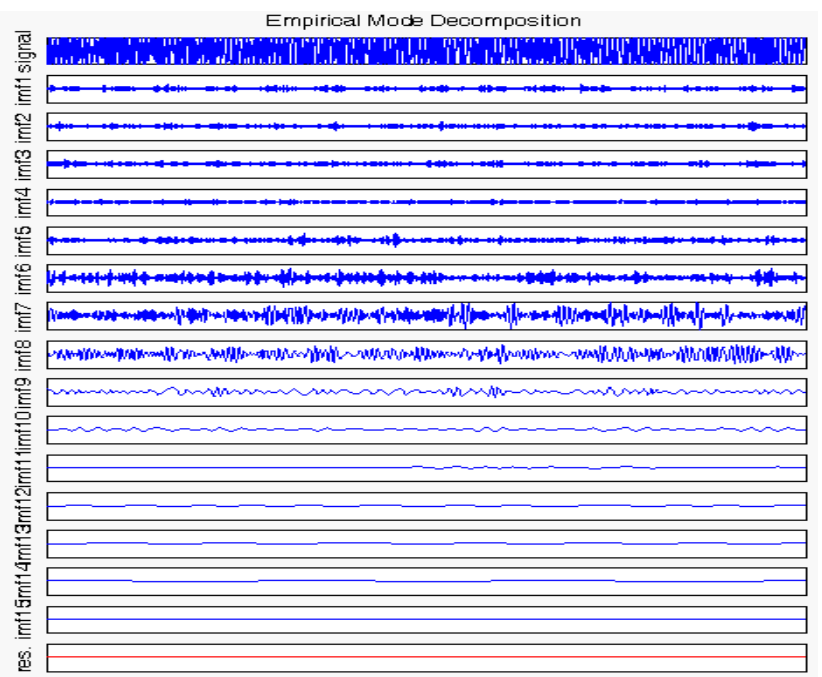

Fig. 3. The various IMFs found in the sound signal of worn tool

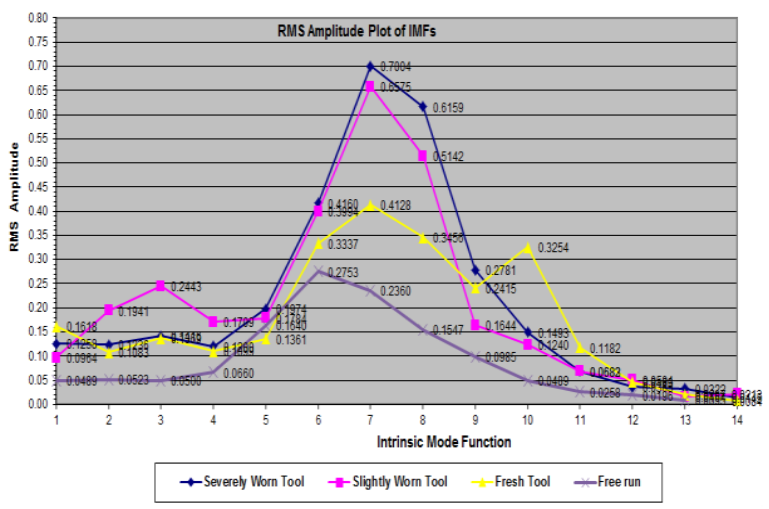

Fig. 4. Comparison of the RMS value of amplitude found in the IMFs of free run, fresh tool, slightly and severely worn tool sound signals.

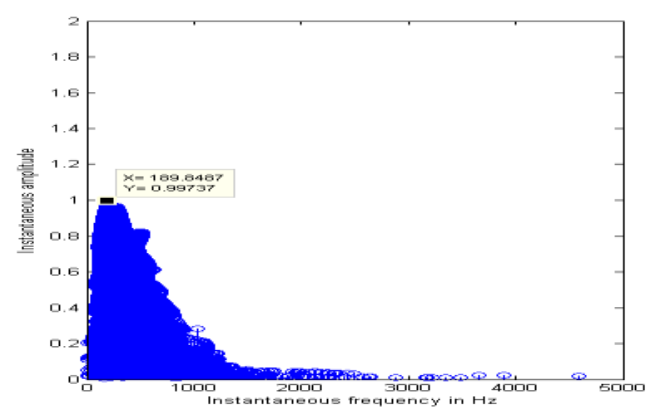

Fig. 5. Marginal spectrum of IMF7 for fresh tool sound signal

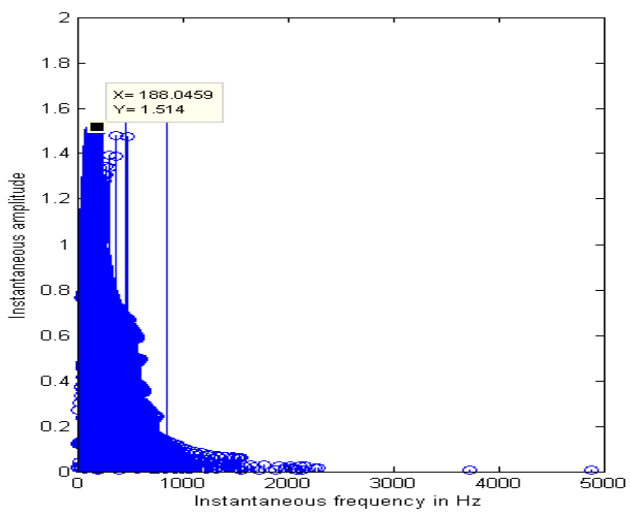

Fig. 6. Marginal spectrum of IMF7 for slightly worn tool sound signals

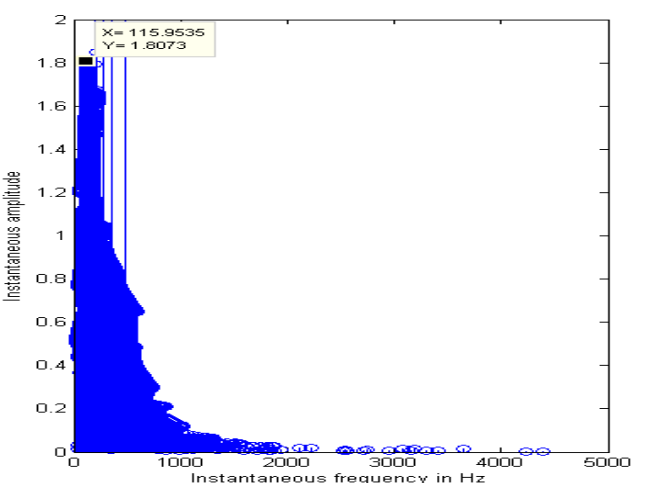

Fig. 7. Marginal spectrum of IMF7 for severely worn tool sound signal

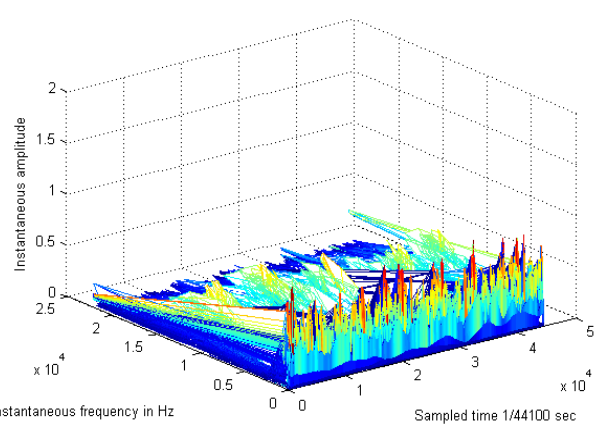

Fig. 8. Hilbert spectrum for fresh tool sound signal

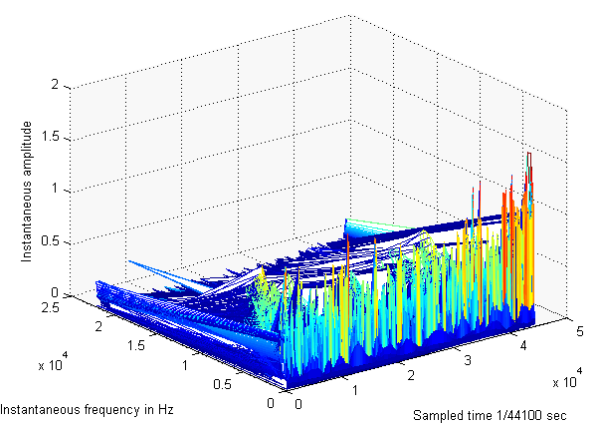

Fig. 9. Hilbert spectrum for slightly worn tool sound signal 


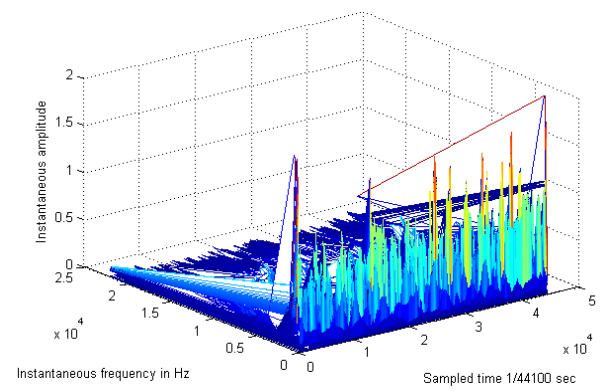

Fig. 10. Hilbert spectrum for severely worn tool sound signal

\section{CONCLUSION}

In this paper, the relationship between the change in the amplitude of the tool emitted sound signal and the tool flank wear was investigated during the turning process of Aluminum work piece. Tool emitted sound signals for three different tool flank wear conditions were experimentally investigated and analyzed using Hilbert-Huang Transform (HHT). It was found that the amplitudes of a few relevant IMF components of the emitted tool sound signal increase with increasing tool flank wear. The results of the investigation confirm that HHT based emitted tool sound signal analysis can be confidently applied to tool flank wear monitoring.

\section{REFERENCES}

[1] B. Sick, "On-line and indirect tool wear monitoring in turning with artificial neural networks: A review of more than a decade of research," Mechanical Systems and Signal Processing, vol. 16(4), pp. 487-546, 2002.

[2] R. G. Silva, R. L. Reuben, K. J. Baker, and S. J. Wilcox, "Tool wear monitoring of turning operations by neural network and expert system classification of a feature set generated from multiple sensors," Mechanical Systems and Signal Processing, vol. 12, pp. 319-332, November 1998.

[3] D. R. Salgado and F. J. Alonso, "An approach based on current and sound signals for in-process tool wear monitoring," International Journal of .Machine Tools and Manufacture, vol. 47, no. 14, pp. 2140-2152, November 2007.

[4] S. Orhan, A. Osman Er, N. Camus-cu, and E. Aslan, "Tool wear evaluation by vibration analysis during end milling of AISI D3 cold work tool steel with 35 HRC hardness," NDT\&E International, vol. 40, pp. 121-126, 2007.

[5] M. Chyuan Lu, E. Kannatey-Asibu, "Analysis of Sound Signal Generation Due to Flank Wear in Turning," Journal of Manufacturing Science and Engineering, vol. 124, no. 4, doi:10.1115/1.1511177, pp. 799-808, November 2002.

[6] F. J. Alonso and D. R. Salgado, "Application of singular spectrum analysis to tool wear detection using sound signals," Proc. IME, Part B: Journal of Engineering Manufacture, vol. 219, no. 9, pp. 703-710, 2005.

[7] A. Samraj, S. Sayeed, J. Emerson Raja, J. Hossen, and A. Rahman, "Dynamic Clustering Estimation of Tool Flank Wear in Turning Process using SVD Models of the Emitted Sound Signals," World Academy of Science, Engineering and Technology, vol. 80, pp. 1322-1326, 2011.

[8] Z. K. Peng, Peter W. Tse, and F. L. Chu, "An improved Hilbert-Hung transform and its application in vibration signal analysis," Journal of Sound and Vibration, vol. 286, pp. 187-205, August 2005.

[9] N. E. Huang, Z. Shen, S. R. Long, M. L. C. Wu, H. H. Shih, Q. N. Zheng, N. C. Yen, C. C. Tung and H.H. Liu, "The empirical mode decomposition and the Hilbert spectrum for nonlinear and non-stationary time series analysis," in Proc. Royal Society of London Series A - Mathematical Physical and Engineering Sciences, 1998, pp. 903-995.

[10] Z. K. Peng, Tse, W. Peter, and F. L. Chu, "A comparison study of improved Hilbert-Huang transform and wavelet transform: Application to fault diagnosis for rolling bearing," Mechanical
Systems and Signal Processing, vol. 19, no. 5, pp. 974-988, September 2001.

[11] L. Sun, M. Shen, and F. H. Y. Chan, "A method for estimating the instantaneous frequency of non-stationary heart sound signals," in Proc. International Conference on Neural Networks and Signal Processing, 2003, vol. 1, pp. 798- 801.

[12] Y. Zhang, "Hilbert-Huang Transform and Marginal Spectrum for Detection of Bearing Localized Defects," in Proc. 6th World Congress on Intelligent Control and Automation, June 21 - 23, 2006, Dalian, China, pp $5457-5461$.

[13] E. C. Titchmarsh, "Introduction to the theory of Fourier integrals," Oxford University Press, 1948, pp. 50-55.

[14] Rilling, G. Flandrin and P. Gonçalv'es, "On Empirical Mode Decomposition and its Algorithms," in Proc. IEEE-EURASIP Workshop on Nonlinear Signal and Image Processing (NSIP2003), 8-11 Jun. 2003, Grado, Italy.

[15] J. Kopac and S. Sali, "Tool wear monitoring during the turning process, Journal of Material Processing and Technology," vol. 113, no. 1-3, pp. 312-316, 2001.

[16] E. Raja Joseph, L. Chu Kiong, L. Way Soong, and S. Purushothaman, "Emitted Sound Amplitude Analysis Using Hilbert Huang Transformation for Cutting Tool Flank Wear Prediction," in Proc. ObCom 2011, Part I, CCIS 269 2012, Springer-Verlag Berlin Heidelberg, 2012, pp. 743-752

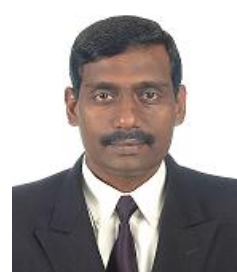

J Emerson Raja received bachelor's and master's degrees in Computer Science and Engineering from University of Madras, India in 1989 and 2001. He is currently pursuing his Ph.D. at Faculty of Engineering and Technology, Multimedia University, Malaysia. His area of research includes machine condition monitoring and soft computing. $\mathrm{He}$ is currently working as a lecturer in Faculty of Engineering and Technology, Multimedia University, Malaysia, since 2005. He was also lecturing in various colleges and universities before coming to this current job. He has published few international journal and conference papers in the area of tool wear monitoring based on emitted sound. He has also co-authored a book, C Programming for beginners, Pearson, Malaysia 2009.

Mr Emerson Raja is an active member of IEEE.

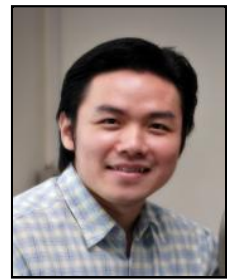

Way-Soong LIM received his Ph.D. degree in Electronic Engineering from Multimedia University of Malaysia, in 2007. He joined the university in 1999 as a lecturer in the Faculty of Engineering \& Technology. Since 2008 , he is serving the faculty as a Deputy Dean and later promoted to Associate Professor in 2010. His research interest covers areas in artificial intelligence, neural network, pattern recognition, condition monitoring and robotics navigation. Along his career path, he has published numerous number of international journals and conference papers, book chapters and engineering text book. Dr. Way-Soong Lim is also a member of IET and IEICE.

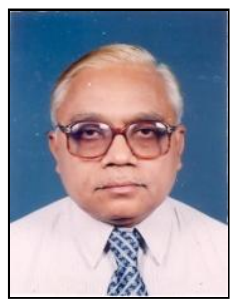

C. Venkataseshaiah received his $\mathrm{PhD}$ degree in Electrical Engineering from Indian Institute of Technology (IIT), Madras in 1976. His major fields of work are Electrical Power Systems, High Voltage Engineering and Instrumentation. He worked in IIT Madras from 1968 to 2005. He was Professor of Electrical Engineering from 1993 to 2005. Since 2006, he is with Faculty of Engineering and Technology, Multimedia University, Melaka campus, Malaysia. He has supervised PhD and Masters Theses at IIT, Madras and MMU. He has published about 85 research papers in international journals and conferences in the areas of Power Systems, High Voltage Engineering, and Instrumentation. He has completed several research projects and held a number of academic and administrative positions at IIT Madras including Chairman, GATE, IIT Madras (1995), Chairman of the Department of Electrical Engineering (2001-2004), Organizing Chairman, National Power Systems Conference (NPSC 2004), India. He was the Chairman of Center for Robotics and Electrical Systems (CRES) at MMU from 2008 to 2011. Dr. C. Venkataseshaiah is a life member of Indian Society for Technical Education (ISTE). He was a member of board of studies and Doctoral and Masters' Thesis Examiner for a few Universities in India and Malaysia. He served as a member of many academic/research committees. He held visiting faculty positions at University of Peradenia (Srilanka) Asian Institute of Technology (Bangkok) and Kwangwoon University (South Korea). 